\title{
ON THE RICCI AND WEINGARTEN MAPS OF A HYPERSURFACE ${ }^{1}$
}

\author{
N. HICKS
}

The purpose of this note is to prove a classical type relation between the Ricci map $R^{*}$ and the Weingarten map $L$ of a hypersurface in a flat Riemannian manifold. Indeed, if $H$ is the mean curvature of the hypersurface, then $L^{2}-H^{\prime} L+R^{*}=0$. This can be viewed, equivalently, as a relation between the Ricci tensor and the second and third fundamental forms. Some obvious corollaries follow.

Let $M$ be an $n$-dimensional $C^{\infty}$ Riemannian manifold, let $X$ and $Y$ be vectors in $M_{m}$, the tangent space at a point $m$ in $M$, and let $R(X, Y)$ be the skew-symmetric linear transformation valued curvature tensor determined by $X$ and $Y$ (see Helgason [2]). We say $M$ is flat if $R \equiv 0$. The Ricci map $R^{*}$ is the self-adjoint linear map $R^{*}(X)=\Sigma R\left(X, Z_{i}\right) Z_{i}$, where $i=1, \cdots, n$ (and this is the standard domain for sum indices in this note), and $Z_{1}, \cdots, Z_{n}$ are an orthonormal base of $M_{m}$. Thus $\operatorname{Ric}(X, Y)=\left\langle R^{*}(X), Y\right\rangle$ gives the symmetric covariant Ricci tensor (this is the negative of the classical Ricci tensor), and $\operatorname{Ric}(X, X)$ is the Ricci curvature of $X$. If $R^{*}$ is a multiple of the identity map at every point of $M$, then $M$ is called an Einstein manifold (see Goldberg $\left[1\right.$, p. 38]). If $R^{*} \equiv 0$ on $M$, then $M$ is Ricci flat. An eigenvector of $R^{*}$ is called a Ricci vector.

We will further assume $M$ is a $C^{\infty}$ hypersurface in a flat $C^{\infty}$ Riemannian manifold $\bar{M}$ of dimension $(n+1)$ and $N$ is a $C^{\infty}$ unit normal vector field on $M$. Let $D$ be the Riemannian covariant differentiation operator on $\bar{M}$. The Weingarten map $L$ is the self-adjoint linear map on each tangent space $M_{m}$ defined by $L(X)=D_{X} N$ (see Hicks [3]). The algebraic invariants of $L$ are the imbedded differential geometric invariants of $M$; in particular, the mean curvature $H=\Sigma k_{i}$ is the trace of $L$ ( $k_{i}$ are the principal curvatures), the second mean curvature $J=\sum_{i<j} k_{i} k_{j}$, and the total curvature $K$ is the determinant of $L$.

For the rest of this note $M$ shall be as described in the above two paragraphs.

TheOREM. $L^{2}-H L+R^{*}=0$.

Proof. We start with the Gauss-curvature equation (see Hicks

Received by the editors January 25, 1964.

1 This work was supported in part by NSF Grant GP-1815. 
[4]) which states that when $X, Y$, and $Z$ are tangent to $M$ at any point $m$ in $M$, then

$$
R(X, Y) Z=\langle L(Y), Z\rangle L(X)-\langle L(X), Z\rangle L(Y) .
$$

Let $Z_{1}, \cdots, Z_{n}$ be an orthonormal base of principal vectors at $m$ with $L\left(Z_{i}\right)=k_{i} Z_{i}$.

Then

$$
\begin{aligned}
R^{*}(X) & =\Sigma R\left(X, Z_{i}\right) Z_{i} \\
& =\Sigma\left[\left\langle k_{i} Z_{i}, Z_{i}\right\rangle L(X)-\left\langle L(X), Z_{i}\right\rangle k_{i} Z_{i}\right] \\
& =H L(X)-\Sigma\left\langle L(X), L\left(Z_{i}\right)\right\rangle Z_{i} \\
& =H L(X)-\Sigma\left\langle L^{2}(X), Z_{i}\right\rangle Z_{i} \\
& =H L(X)-L^{2}(X)
\end{aligned}
$$

since $L$ is self-adjoint.

COROLlaRy 1. Every principal vector $X$ is a Ricci vector.

COROLLARY 2. Every surface in three space is an Einstein manifold.

Proof. If $n=2$, the characteristic equation for $L$ is $L^{2}-H L+K I$ $=0$, where $I$ is the identity map, so $R^{*}=K I$.

CoROllary 3. If the Ricci curvature of every vector on $M$ is nonpositive, then the second mean curvature is nonpositive on $M$.

Proof. Let $X$ be a unit principal vector with $L(X)=k X$. Then $\operatorname{Ric}(X, X)=\left\langle R^{*}(X), X\right\rangle=k H-k^{2} \leqq 0$. Thus, for each principal curvature $k_{i}, \sum_{j=1, j \neq i}^{n} k_{i} k_{j} \leqq 0$, which implies $\sum_{i<j} k_{i} k_{j}=J \leqq 0$.

COROLLARY 4. On a minimal hypersurface the Ricci curvature of every vector is nonpositive and hence the second mean curvature is nonpositive.

Proof. In this case, $\operatorname{Ric}(X, X)=\left\langle R^{*}(X), X\right\rangle=\left\langle-L^{2}(X), X\right\rangle$ $=-\langle L(X), L(X)\rangle \leqq 0$.

COROLlaRy 5. An Einstein hypersurface has at most two distinct principal curvatures.

Proof. If $R^{*}=b I$, then every principal curvature must satisfy the equation $k^{2}-H k+b=0$.

CoRollary 6. A hypersurface is Ricciflat if and only if it is Einstein with total curvature zero. If $n=3$ and $M$ is Ricci flat, then the second mean curvature is also zero so at points $m$ on $M$ that are not flat points $\left(L_{m} \neq 0\right)$, the multiplicity of the nonzero principal curvature is unity. 
Proof. If $M$ is Ricci flat it is trivially Einstein and, since $L^{2}-H L$ $=0$, one of the principal curvatures is zero.

If $R^{*}=b I$, then $L^{2}-H L+b I=0$. If $K=0$, then there is a zero principal curvature and a unit principal vector $X$ with $L X=0$. Hence $b X=0$ so $R^{*}=0$.

In the case $n=3$, the characteristic polynomial $L^{8}-H L^{2}+J L-K I$ $=0$ implies $J L=0$, and since $L_{m}=0$ implies $J(m)=0$, we have $J \equiv 0$.

\section{BIBLIOGRAPHY}

1. S. Goldberg, Curvature and homology, Academic Press, New York, 1962.

2. S. Helgason, Differential geometry and symmetric spaces, Academic Press, New York, 1962.

3. N. Hicks, Connexion preserving, conformal, and parallel maps, Michigan Math. J. 10 (1963), 295-302.

4. - Submanifolds of semi-Riemannian manifolds, Rend. Circ. Mat. Palermo 12 (1964), 1-13.

UNIVERSITY OF MichigAN

\section{ON PSEUDOMETRICS FOR GENERALIZED UNIFORM STRUCTURES}

\section{SOLOMON LEADER}

In [1] Alfsen and $\mathrm{Njåstad} \mathrm{generalized} \mathrm{the} \mathrm{concept} \mathrm{of} \mathrm{a} \mathrm{uniform}$ structure $\mathcal{U}$ on a set $S$, replacing the intersection axiom for uniform structures by the weaker condition:

(0) Given subsets $A_{1}, \cdots, A_{n}$ of $S$ and $U_{1}, \cdots, U_{n}$ in $\mathcal{u}$, there exists $U$ in $u$ such that $U\left(A_{i}\right) \subseteq U_{i}\left(A_{i}\right)$ for $i=1, \cdots, n$. Our object is to characterize these structures in terms of pseudometrics.

Define a (generalized) gage on $S$ to be a nonvoid family $\mathcal{G}$ of pseudometrics on $S \times S$ such that

(1) Every pseudometric uniformly continuous with respect to $\mathcal{G}$ belongs to $\mathrm{g}$.

(2) If $\alpha$ and $\beta$ belong to $\mathcal{G}$ and both $\alpha$ and $\beta$ are totally bounded, then $\alpha \bigvee \beta$ belongs to $\mathrm{g}$.

Note that if we delete the total boundedness condition in (2), then $\mathcal{G}$ is just a gage for a proper uniform structure [2], [3]. For $\beta$ a pseudometric on $S \times S$, define $W_{\beta}=\beta^{-1}[0,1)$.

TheOREM. Given a gage $\mathcal{G}$ on $S$, define the class $\mathcal{U}$ of subsets $U$ of $S \times S$ by the condition

Received by the editors January 28, 1964. 